\title{
ESTIMATION DES CARACTÉRISTIQUES BIOLOGIQUES DES TRUITES DE MER ADULTES (SALMO TRUTTA) DU RHIN SUPÉRIEUR.
}

\author{
D. OMBREDANE (1, 2), P. ROCHE (3), J.L. BAGLiNIÈRE (2), G. EDEL (4), \\ M. GERLIER (4) et B. GIPPET $(1,2)$
}

(1) E.N.S.A.R., Département Environnement et Exploitation des Ressources Naturelles, 65 rue de Saint-Brieuc, 35042 Rennes Cedex, France.

(2) INRA, Laboratoire d'Ecologie Aquatique, 65 rue de Saint-Brieuc, 35042 Rennes Cedex, France.

(3) Conseil Supérieur de la Pêche, Délégation Champagne-Ardennes-Lorraine-Alsace, 18 rue Nomény, 57950 Montigny-les-Metz, France.

(4) Association Saumon-Rhin, Tour du Schloessel, 33 rue de la Tour, 67200 Strasbourg, France.

\section{RÉSUMÉ}

L'analyse des longueurs totales et des écailles d'un échantillon de 120 truites de mer adultes capturées essentiellement par pêche à l'électricité dans le Rhin supérieur $(700 \mathrm{~km}$ de la mer du Nord) de 1989 à 1996 a permis de caractériser la population de cette ressource piscicole en voie de restauration. L'utilisation des Captures Par Unité d'Effort (CPUE), calculées pour les pêches de 1994 à 1996, permet d'appréhender le rythme de migration et d'améliorer l'estimation des caractéristiques générales de la population.

Dans cette partie du Rhin, la migration anadrome de la truite de mer se déroule en deux pics successifs de juin à décembre, les individus les plus âgés arrivant les premiers.

La population est caractérisée par une très forte prédominance des groupes d'âge de mer $1+(49,2 \%)$ et $2+(45,8 \%)$, soit un Age Moyen de Mer (AMM) de 1,54 an. L'Age Moyen de Smoltification (AMS) et de première Reproduction (AMR) sont estimés respectivement à 1,30 an et 1,39 an. Par ailleurs, il est constaté que l'âge marin de première maturité des adultes du Rhin augmente quand leur âge de smoltification diminue.

Les résultats sont discutés en fonction de la méthodologie employée et comparés à ceux obtenus sur d'autres populations françaises et plus nordiques. II ressort que les caractéristiques de la population de truite de mer du Rhin traduisent bien la position latitudinale mais aussi l'importance de ce réseau hydrographique.

\section{ESTIMATION OF BIOLOGICAL CHARACTERISTICS OF ADULT SEA TROUT (SALMO TRUTTA) IN THE UPPER RHINE.}

\author{
ABSTRACT \\ An analysis of the total length and scales of a sample of 120 adult sea trout caught \\ for the most part by electrofishing in the upper Rhine (700 km from the North Sea) from 1989
}


to 1996 lent information to characterize this population under restoration. The calculation of the Catch Per Unit of Effort (CPUE) with data from 1994 to 1996 allowed to study the migration pattern and to improve the estimation of the general population characteristics.

In this part of the Rhine, anadromous migration of sea trout occurs with two successive peaks from June to December, the older fish arriving first.

The population is characterized by a predominance of fish aged $1+(49,2 \%)$ and $2+$ $(45,8 \%)$ years at sea, that is to say an average sea age (AMM) of 1.54 year. The average smolting age (AMS) and sea age at maturity (AMR) are respectively estimated at 1.30 and 1.39 year. Moreover, in this population, sea age at maturity increases when smolt age decreases.

The results are discussed according to the methods and are compared with results obtained from other French or more northern rivers populations. Thus, the characteristics of the Rhine sea trout population correspond to what could be expected from its latitudinal situation and its great river status.

\section{INTRODUCTION}

Le saumon atlantique (Salmo salar) et la truite de mer (Salmo trutta) font l'objet depuis le début des années 1990 d'un programme de restauration dans le bassin du Rhin, programme dénommé "saumon 2000 » (ANONYME, 1994). Contrairement au saumon dont la réapparition en 1990 dans le Rhin moyen puis en 1995 dans le Rhin supérieur résulte d'une réintroduction (STEINBERG et al., 1991 ; ROCHE et al., 1996), la forme migratrice marine de la truite (truite de mer) a reconquis le Rhin et la Meuse vers la fin des années 1970, 10 ans avant l'intervention du programme de restauration (PHILIPPART, 1983 ; OLBRICH, 1984 ; WEIBEL, 1991 ; STEINBERG et LUBIENIECKI, 1991). Cette réapparition correspond à une période d'amélioration très nette de la qualité de l'eau, en particulier en ce qui concerne l'oxygène dissous (CAZEMIER, 1988). La truite de mer n'avait cependant pas complètement disparu de l'estuaire du Rhin si l'on en croit les déclarations de prises faites aux Pays-Bas, qui se situaient aux environs de 10 tonnes par an pendant les années de pollution intense qu'a connues le fleuve, entre 1950 et 1980 (GROOT (de), 1989 ; CAZEMIER, 1992). En partie française du bassin du Rhin supérieur, dans un secteur situé à environ $700 \mathrm{~km}$ de la mer, les pêcheurs à la ligne ont été les premiers à signaler vers la fin des années 1970 de grandes truites présentant les caractères morphologiques de la forme marine. L'étude de la structure et de la teneur en strontium des écailles de 26 poissons capturés principalement à la ligne ont confirmé qu'il s'agissait bien, pour la plupart d'entre elles, de truites de mer (ROCHE, 1992).

Dans le cadre du programme " saumon 2000 ", des pêches à l'électricité effectuées depuis 1992 ont permis des captures plus nombreuses de truites de mer. Même si les données récoltées à partir de ces échantillonnages n'ont pas le caractère exhaustif de celles obtenues par des systèmes de piégeage en continu, elles permettent néanmoins d'approcher quelques caractéristiques biologiques et écologiques de la population d'adultes de truite de mer du Rhin supérieur. Ces caractéristiques peuvent alors être comparées à celles d'autres systèmes hydrographiques européens.

\section{MATÉRIEL ET MÉTHODE}

\subsection{Site d'étude}

L'étude a eu lieu dans le Rhin supérieur franco-allemand, principalement au niveau des barrages d'Iffezheim et de Gambsheim de structure identique et situés respectivement 
à 689 km (pk 334) et 714 km (pk 309) de la mer du Nord (Figure 1). Le barrage d'lffezheim est le premier obstacle du cours principal du Rhin sur le trajet des salmonidés migrateurs vers leurs frayères du Rhin supérieur. Le débit moyen interannuel au niveau des deux barrages est de l'ordre de $1100 \mathrm{~m}^{3} \mathrm{~s}^{-1}$.

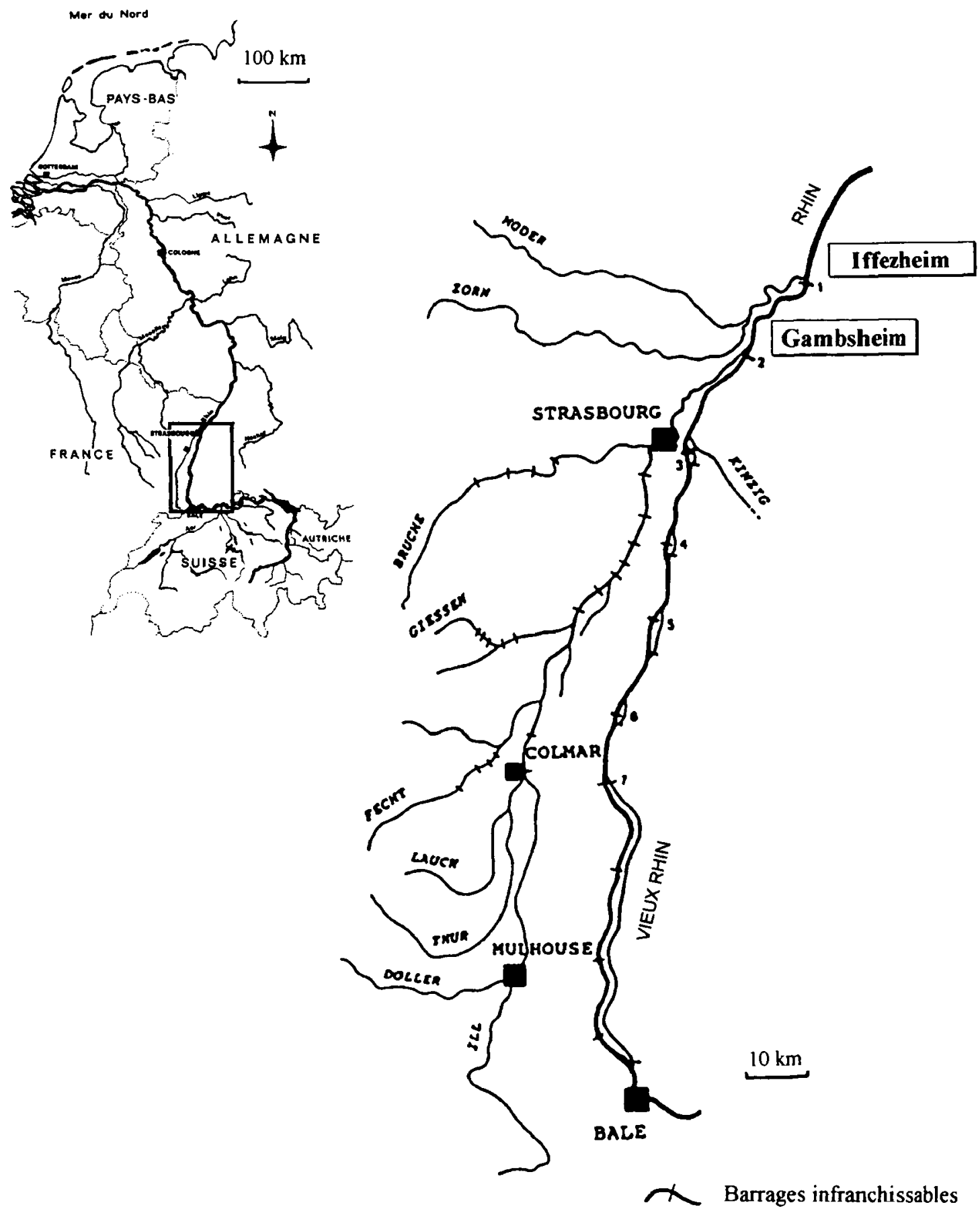

Figure 1

Localisation des sites d'étude sur le Rhin supérieur.

Figure 1

Map of the study sites in the upper Rhine. 


\subsection{Méthodes de capture et données disponibles}

Depuis 1992, des pêches à l'électricité en bateau sont réalisées à l'aval immédiat des deux barrages. Les salmonidés capturés ont été conservés pour leur reproduction en pisciculture (1992 à 1995) ou relâchés après marquage en amont des barrages (1996).

Les pêches ont été effectuées avec deux anodes plongeantes, l'une lancée 3 à $5 \mathrm{~m}$ à l'avant du bateau, l'autre pêchant près de la rive. La profondeur maximale atteinte était de l'ordre de 1,5 $\mathrm{m}$. Lors de chaque pêche, les deux rives des canaux de fuite des usines ont été prospectées $(2,4 \mathrm{~km}$ à Iffezheim, $2 \mathrm{~km}$ à Gambsheim, temps de pêche : $1 \mathrm{~h} 30 \mathrm{~min}$. environ), ainsi que l'aval du barrage de régulation d'lffezheim lorsque celui-ci déverse $(0,3 \mathrm{~km}$, temps de pêche : $15 \mathrm{~min}$. environ). Lorsque plusieurs truites ou saumons étaient capturés au cours d'une sortie, le temps de pêche a été généralement un peu plus élevé (plus 10 à $20 \%$ ) car l'aval immédiat de la centrale électrique est prospecté 2 à 3 fois. Le temps de pêche n'a pas toujours été enregistré avec précision mais il est resté malgré tout peu variable durant la période d'étude. Aussi, l'unité d'effort retenue est la sortie de pêche.

Les dates et efforts de pêche ainsi que les nombres de captures par sortie ne sont précisément connus que pour les années 1994, 1995 et 1996. La fréquence des pêches a varié d'une année à l'autre, mais aussi d'un mois à l'autre car elle est souvent fonction du nombre de salmonidés capturés (Figure 2a). Par exemple, en août et septembre, les pêches sont plus espacées car les captures sont souvent plus rares (Figure 2b), sauf en cas de crue tardive du Rhin.

Afin d'augmenter la taille de l'échantillon de poissons analysés, sont aussi pris en compte 30 individus capturés par pêche à la ligne depuis 1989 dans le réseau rhénan supérieur (Rhin, Moder, Ill...) dont ceux étudiés par ROCHE (1992). L'échantillon de base est donc composé de 142 truites adultes.

\subsection{Caractéristiques des individus}

\section{Mesures au moment de la capture}

Après avoir été anesthésiés, les salmonidés ont été mesurés (longueur totale) et un échantillon d'écailles a été prélevé. Les caractéristiques biométriques n'ont été notées systématiquement au moment de la capture qu'à partir de 1996. Les années précédentes, ces données n'ont été relevées que sur les poissons survivants et relâchés après la reproduction artificielle à la pisciculture de la Fédération de Pêche du Bas-Rhin, ceci afin de limiter le stress avant le transfert en pisciculture. Les écailles et les longueurs ne sont donc disponibles que pour une partie des captures, variable elle aussi d'une année sur l'autre (respectivement $50 \%, 14 \%$ et $100 \%$ des captures en 1994, 1995 et 1996).

En se basant sur la livrée du poisson, l'espèce et la forme de truite sont notées:

- TRM : Truite de mer, forme migratrice marine de Salmo trutta,

- TRF : Truite fario, forme résidente (eau douce) de Salmo trutta,

- TAC : Truite arc-en-ciel (Oncorhynchus mykiss).

\section{Interprétation des écailles}

L'examen de deux à une dizaine d'écailles par truite a permis dans un premier temps de valider la séparation des individus par espèce (forme générale, structures fines ; BAGLINIĖRE et LE LOUARN, 1987), puis par forme pour l'espèce Salmo trutta. Pour cette distinction a posteriori des deux formes de truite, les écailles dont l'interprétation avait été confortée par une analyse de leur teneur en strontium (ROCHE, 1992) ont servi de 


\section{a : EFFORTS}

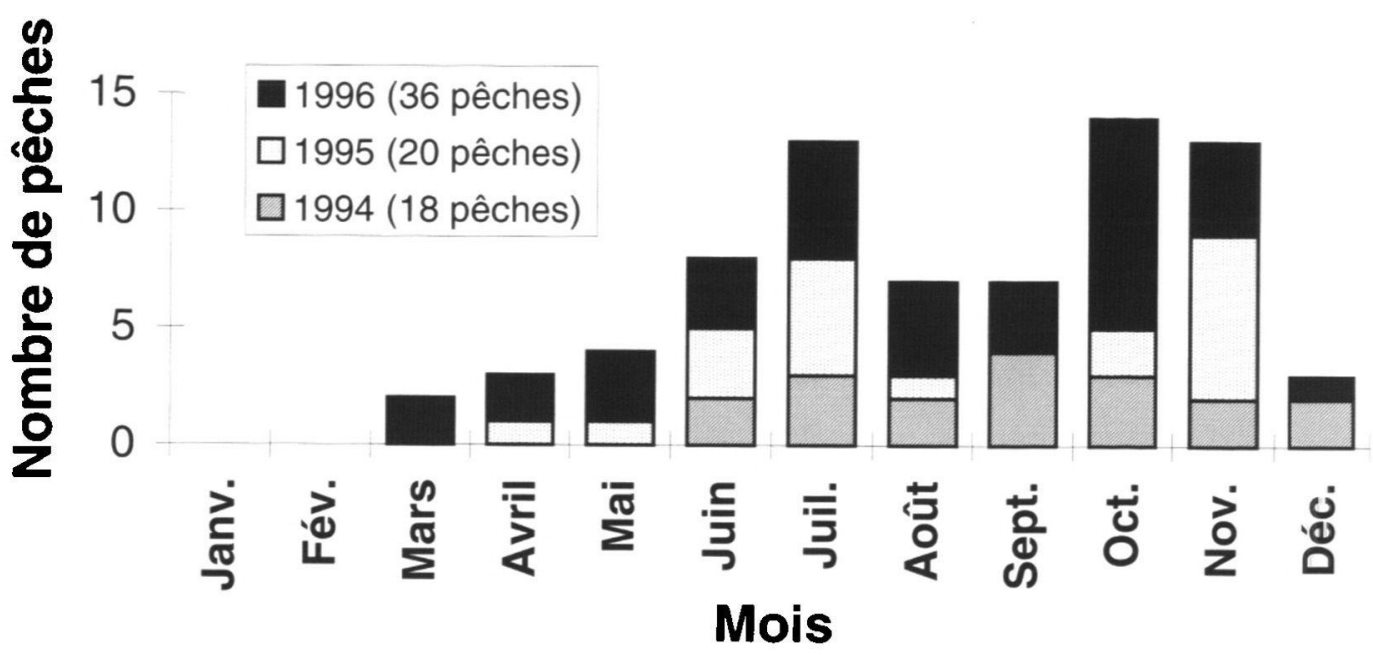

\section{$b$ : CAPTURES}

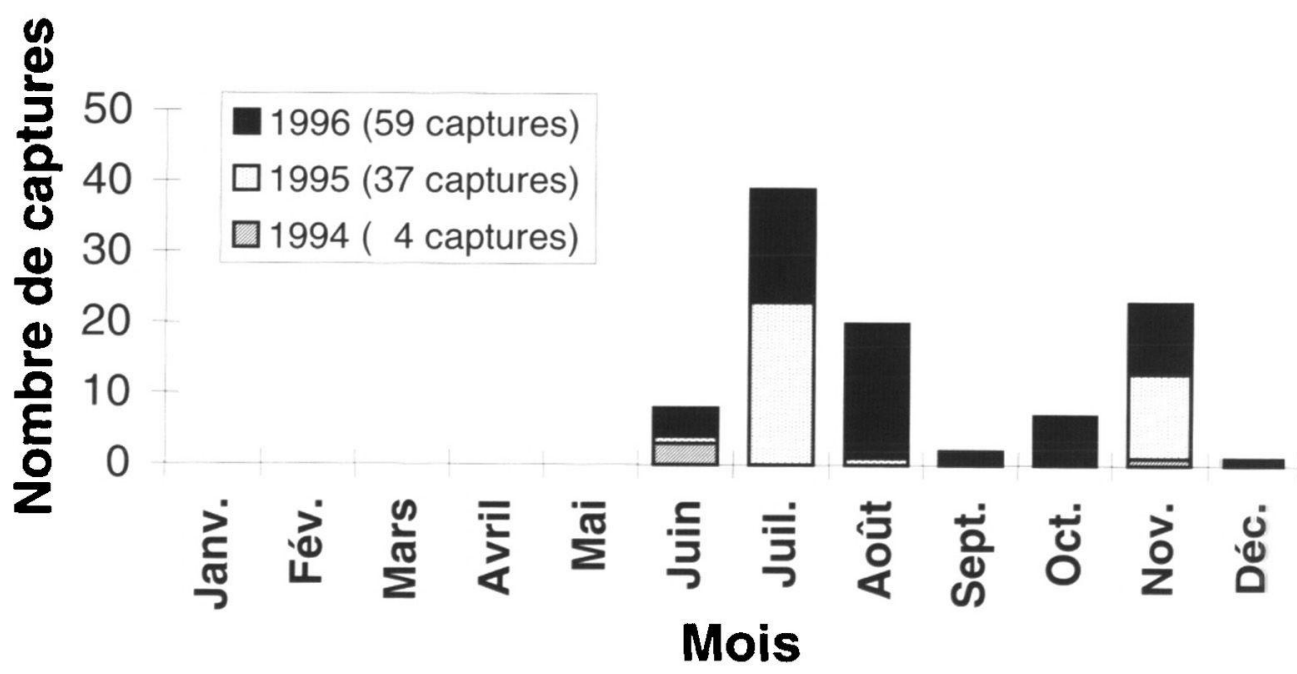

Figure 2

Efforts de pêche (a) et Captures (b) de truites de mer en aval du barrage d'Iffezheim de 1994 à 1996.

Figure 2

Fishing efforts (a) and Catches (b) of sea trout below Iffezheim dam from 1994 to 1996.

référence. Dans un second temps, l'analyse scalimétrique a permis d'estimer les âges d'eau douce, de mer et de première reproduction des truites de mer du genre Salmo. Ces estimations ont été faites en utilisant les critères d'interprétation et la notation préconisés par RICHARD (1986). 
Les truites de mer sont alors classées en plusieurs types selon la durée de leur séjour marin avant la première reproduction (RICHARD, 1986) :

- Poisson de type $0: 3$ à 6 mois ( Finnock"),

- Poisson de type I : 1 hiver, soit une durée de 13-18 mois,

- Poisson de type II : 2 hivers, soit une durée de 25-30 mois,

- Poisson de type III : 3 hivers, soit une durée de 35-40 mois.

Compte tenu du phénomène important de régénération des écailles chez la truite (OMBREDANE et RICHARD, 1990), ces différents critères n'ont pas pu être estimés sur la totalité de l'échantillon de base de 142 individus :

- l'espèce et la forme n'ont été validées que sur 140 individus parmi lesquels ont été identifiées 122 truites de mer,

- parmi ces 122 truites de mer, l'âge d'eau douce et l'âge de mer n'ont pu être estimés que sur respectivement 111 et 120 individus,

- il faut aussi noter que parmi ces 120 truites de mer adultes, les dates de capture n'étaient connues précisément que pour 118 individus (respectivement 11, 12, 8, 22, 3, 5, 57 en 1989, 1990, 1992, 1993, 1994, 1995, 1996).

\subsection{Caractéristiques de la population}

Afin de caractériser la population d'un cours d'eau et de pouvoir la comparer à celles d'autres réseaux hydrographiques, différents indices peuvent être calculés : un Age Moyen de Smoltification (AMS ; FAHY, 1978), un Age Moyen Marin (AMM) et un Age de mer Moyen à la première Reproduction (AMR) (RICHARD, 1986). Ces âges moyens sont classiquement calculés avec les données récoltées sur l'échantillon de poissons capturés. Mais ils ne peuvent caractériser la population d'un cours d'eau que dans la mesure où l'échantillon des individus est représentatif, à savoir quand l'échantillonnage est systématique ou correctement stratifié (LE CREN, 1985), avec des efforts de capture et une périodicité régulière notamment. Dans le cas présent, l'échantillon de truites capturées ne satisfaisant pas à ces conditions, un autre mode de calcul prenant en compte les captures par unité d'effort mensuelles a aussi été utilisé pour l'estimation de ces âges moyens.

Soit pour une population de truites de mer en migration anadrome capturées en un lieu donné :

$A=$ Age moyen de la population (AMS, AMM ou AMR),

$n_{i, j}=$ stock arrivant le mois $i$ et d'âge $j$,

$\mathrm{N}_{\mathrm{i}}=\Sigma \mathrm{n} \mathrm{i}, \mathrm{j}=$ stock arrivant le mois $\mathrm{i}$ tous âges confondus,

$\mathrm{N}_{\mathrm{j}}=\Sigma \mathrm{n}_{\mathrm{i}, \mathrm{j}}=$ stock d'âge $\mathrm{j}$ tous mois confondus,

$\mathrm{N}=\Sigma \mathrm{Ni}_{\mathrm{i}}=$ population totale,

$c_{i, j}=$ captures du mois $i$ et d'âge $j$,

$\mathrm{C}_{\mathrm{i}}=\Sigma \mathrm{C}_{\mathrm{i}, \mathrm{j}}=$ captures du mois $\mathrm{i}$ tous âges confondus,

$C_{j}=\Sigma c_{i, j}=$ captures d'âge $j$ tous mois confondus,

$\mathrm{C}=\Sigma \mathrm{C}_{\mathrm{i}}=$ captures totales,

$\mathrm{u}_{\mathrm{i}}=\mathrm{C}_{\mathrm{i}} / \mathrm{N}_{\mathrm{i}}=$ taux de sondage de la population le mois $\mathrm{i}$,

$\mathrm{u}=\mathrm{C} / \mathrm{N}=$ taux de sondage de la population totale,

$\mathrm{f}_{\mathrm{i}}=$ effort de pêche du mois $\mathrm{i}=$ nombre de sorties de pêche le mois $\mathrm{i}$,

$\mathrm{q}_{\mathrm{i}}=$ capturabilité des poissons lors des pêches du mois $\mathrm{i}$,

CPUE $_{i}=\mathrm{Ci} / f_{i}=$ Captures Par Unité d'Effort le mois $\mathrm{i}$. 


\section{Caractéristiques de l'échantillon}

Sur la base des échantillons de truites de mer dont les âges ont pu être estimés, les âges moyens A ont été calculés ainsi (d'après FAHY, 1978 ; RICHARD, 1986) :

$$
A=\left[\Sigma\left(C_{j} \times j\right)\right] /\left[\Sigma C_{j}\right]
$$

- avec $C_{j}=$ le nombre de poissons d'âge d'eau douce j pour l'AMS,

- avec $C_{j}=$ le nombre de poissons des différents âges de mer $\mathrm{j}$ à la capture pour l'AMM,

- avec $C_{j}=$ le nombre de poissons s'étant reproduits pour la première fois après avoir passé j hivers en mer pour l'AMR.

La variance des âges dans l'échantillon a été estimée :

$$
\hat{\sigma^{2}}=\left[\Sigma C_{j}(j-A)^{2}\right] /\left[\left(\Sigma C_{j}\right)-1\right]
$$

Les captures ayant été réalisées selon un échantillonnage aléatoire simple, la variance de l'âge moyen Var (A) s'exprime ainsi (SCHERRER, 1983) :

$$
\operatorname{Var}(A)=\left[\hat{\sigma^{2}} /\left(\Sigma C_{j}\right)\right][1-u]
$$

Les données disponibles ne permettent pas d'estimer $u$, mais comme ce paramètre est très vraisemblablement très faible, $\operatorname{Var}(\mathrm{A})$ a été majorée par :

$$
\operatorname{Var}(A) \leq\left[\hat{\sigma^{2}} /\left(\Sigma C_{j}\right)\right]
$$

\section{Caractéristiques de la population}

Pour évaluer les âges moyens de la population, il a été considéré que les poissons avaient été capturés selon un protocole pouvant s'apparenter à un échantillonnage aléatoire simple stratifié, chaque mois étant considéré comme une strate.

L'âge moyen du stock arrivant le mois $\mathrm{i}, \mathrm{A}_{\mathrm{i}}$ s'exprime par la formule suivante :

$$
A_{i}=\Sigma\left(n_{i, j} \times j\right) / N_{i}
$$

Aussi l'âge moyen de la population $A$ peut s'exprimer en fonction des $A_{i}$ :

$$
A=\left[\Sigma\left(\Sigma n_{i, j} \times j\right)\right] / N=\left[\Sigma\left(A_{i} \times N_{i}\right)\right] /\left[\Sigma N_{i}\right]
$$

Afin que les captures et les CPUE mensuelles puissent être considérées comme représentatives (qualitativement et quantitativement) du stock arrivant chaque mois, deux hypothèses ont été admises :

1- la capturabilité q est constante quelle que soit la période et quels que soient l'âge, la taille du poisson et l'état physiologique du poisson,

2- les poissons non capturés ne stagnent pas plus d'un mois au pied du barrage (hypothèse qui n'est pas nécessaire si la capturabilité est élevée).

Dans ces conditions, il est alors possible d'estimer $A_{i}$ :

$$
\hat{A}_{i}=\left(\Sigma c_{i, j} \times j\right) / C_{i}
$$


Par ailleurs, la CPUE peut être considérée comme un indice d'abondance pour une période donnée (LAUREC et LE GUEN, 1981) :

$$
\mathrm{C}_{\mathrm{i}} / \mathrm{f}_{\mathrm{i}}=\mathrm{q}_{\mathrm{i}} \times \mathrm{Ni}_{\mathrm{i}}
$$

soit :

$$
N_{i}=1 / q_{i} \times C P U E_{i}
$$

A partir des formules (a), (b) et (c) on peut alors exprimer $\hat{A}$ par :

$$
\hat{A}=\left[\Sigma\left(\hat{A}_{i} \times \operatorname{CPUE}_{i} / q_{i}\right)\right] /\left[\Sigma \operatorname{CPUE}_{i} / q_{i}\right]
$$

Les capturabilités mensuelles qi n'étant pas connues, la sensibilité de l'expression (d) à ce paramètre a été testée par une procédure de Monte Carlo. La fonction ALEA du logiciel Excel a permis de générer des valeurs aléatoires de qi distribués selon une loi uniforme et comprises entre 0,05 et 0,5 (gamme plausible de capturabilité). Pour 1000 itérations, les $\hat{A}$ obtenus ont une distribution presque normale et le coefficient de variation de leur moyenne est inférieur à $5 \%$, ce qui traduit une faible sensibilité du modèle à qi. Aussi les capturabilités qi ont toutes été considérées comme égales à q quel que soit le mois. L'expression (d) se simplifie et l'âge moyen de la population peut être estimé par :

$$
\hat{A}=\left[\Sigma\left(\hat{A}_{i} \times C P U E_{i}\right)\right] /\left[\Sigma \text { CPUE }_{i}\right]
$$

$\operatorname{avec} \operatorname{SCHERRER}, 1983: \operatorname{Var}(\hat{A})=\Sigma\left\{\left[\left(\mathrm{CPUE}_{\mathrm{i}} / \Sigma \mathrm{CPUE}_{\mathrm{i}}\right)^{2} \times \hat{\sigma}_{i}^{2} /\left(\Sigma \mathrm{C}_{\mathrm{j}}\right)\right]\left[1-\mathrm{u}_{\mathrm{i}}\right]\right\}$

De même, comme les ui ne sont pas connus, $\operatorname{Var}(\hat{A})$ a été majoré par :

$$
\operatorname{Var}(\hat{A}) \leq \Sigma\left[\left(\text { CPUE }_{i} / \Sigma \mathrm{CPUE}_{\mathrm{i}}\right)^{2} \times \hat{\sigma}_{\mathrm{i}}^{2} /\left(\Sigma \mathrm{C}_{\mathrm{j}}\right)\right]
$$

Pour cette étude, les différents âges moyens de la population ont donc été estimés à partir des âges moyens mensuels de l'échantillon pour lesquels les âges et les dates de capture étaient connus, toutes années de capture confondues. De plus, ont été utilisées les CPUE mensuelles moyennes des années 1994 à 1996 à Iffezheim.

Le rythme de migration anadrome des truites de mer a été étudié par l'analyse de la variabilité des CPUE mensuelles à Iffezheim.

\section{RÉSULTATS}

\subsection{Rythme de migration}

Les CPUE mensuelles sont variables d'un mois à l'autre mais aussi d'une année à l'autre pour un mois donné (Figure 3a). Notamment, elles sont fréquemment nulles en 1994. Cependant, les évolutions mensuelles montrent une même tendance générale qui se dégage plus nettement en cumulant les captures et les efforts des trois années pour le calcul des CPUE mensuelles (Figure 3b). Les premières captures de truites de mer ont lieu en juin dans le Rhin supérieur et augmentent jusqu'au milieu de l'été (juillet-août). Puis, les CPUE diminuent fortement au début de l'automne (septembre-octobre) pour augmenter de nouveau en novembre, ce qui se traduit par un second pic d'abondance mais moins important que le premier. 
a

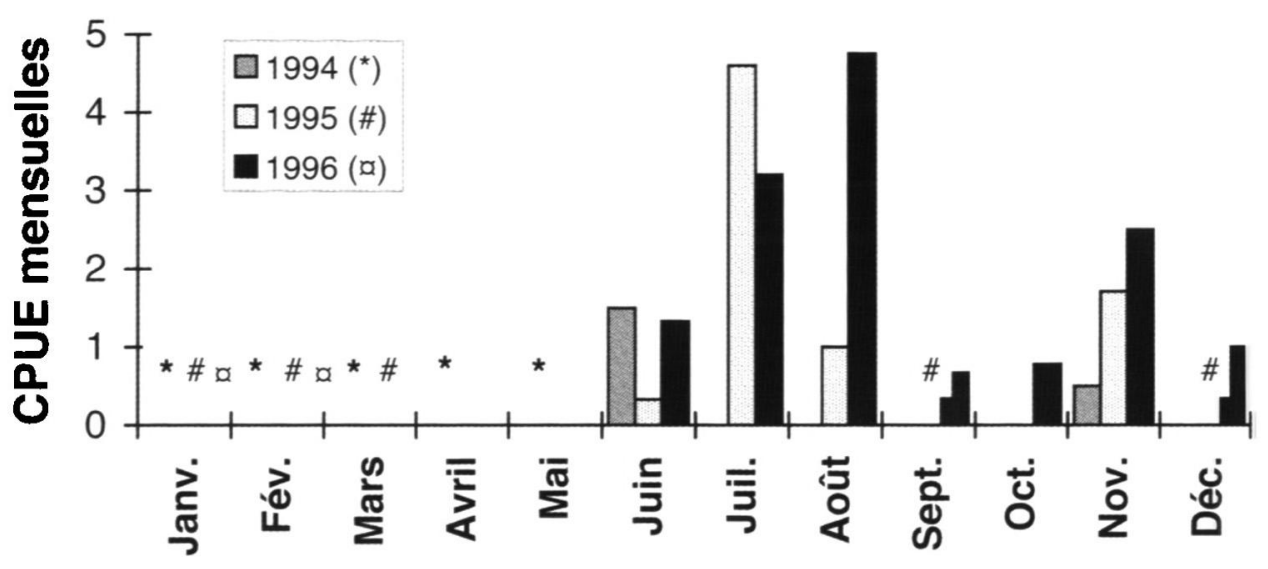

Mois

b : CPUE moyennes $1994-1996$

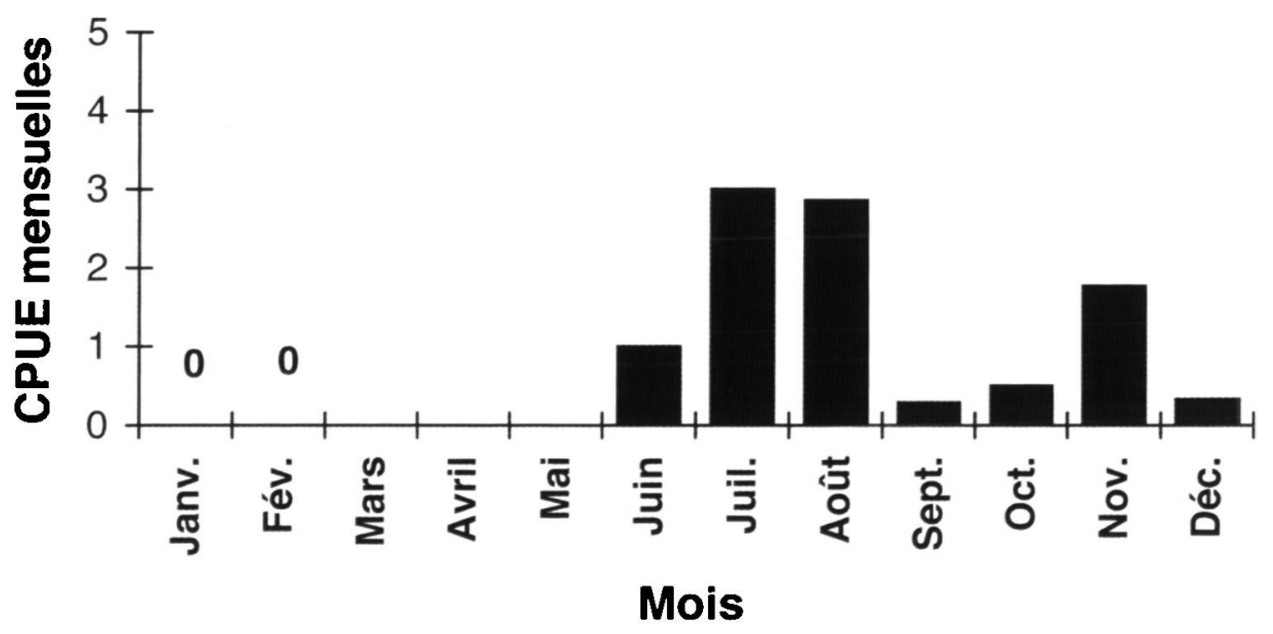

$$
\text { *,\#, }, 0 \text { = effort de pêche nul }
$$

\section{Figure 3}

Rythme de migration anadrome des adultes de truite de mer dans le Rhin supérieur ( $a$ = Captures par unité d'effort de 1994, 1995 et 1996 - b = Captures par unité d'effort moyennes 1994-1996).

\section{Figure 3}

Sea trout anadromous migration patterns in the upper Rhine ( $a=$ Catches per unit of effort in 1994, 1995 and $1996-b=$ Average catches per unit of effort for 1994-1996). 


\subsection{Caractéristiques démographiques}

\section{Age d'eau douce}

Toutes les truites de mer de l'échantillon (111 individus) n'ont séjourné que 1 ou 2 ans en rivière, la première classe d'âge étant largement majoritaire $(68,5 \%$ contre $31,5 \%)$.

L'âge moyen d'eau douce (AMS) de l'échantillon est de 1,32 an et celui de la population, calculé à partir des CPUE, de 1,30 an avec des écarts types des moyennes calculées respectivement de 0,04 et 0,05 pour les deux méthodes.

La longueur totale moyenne des truites ayant smoltifié à 1 an $(617 \mathrm{~mm}, \sigma=79)$ n'est pas statistiquement différente (Test $t, P=0,19$ ) de celle des poissons ayant smoltifié à 2 ans (594 $\mathrm{mm}, \sigma=98$ ), tous âges de mer confondus. Ce même phénomène est statistiquement mis en évidence pour chaque âge de mer analysé séparément (Tableau I).

\section{Tableau I}

Caractéristiques biométriques et démographiques d'un échantillon de 120 adultes de truite de mer capturés dans le Rhin supérieur ( $\mathrm{i}$ = âge d'eau douce indéterminé, Lt $=$ Longueur totale).

\section{Table I}

Biometric and demographic characteristics of a sample of 120 adult sea trout captured in the upper Rhine ( $i=$ freshwater age unknown, $L t=$ total length).

\begin{tabular}{|c|c|c|c|c|c|}
\hline Age de mer & $\begin{array}{l}\text { Age d'eau } \\
\text { douce }\end{array}$ & $\begin{array}{l}\text { Lt moyenne } \\
(\mathrm{mm})\end{array}$ & $\begin{array}{c}\text { Lt } \\
\text { écart type }\end{array}$ & Effectifs & $\begin{array}{c}\% \text { de la } \\
\text { population }\end{array}$ \\
\hline 0 & 1 & 468 & -- & 1 & 0,8 \\
\hline \multirow[t]{4}{*}{1} & $\mathbf{i}$ & 544 & 33 & 4 & 3,3 \\
\hline & 1 & 547 & 46 & 30 & 25,0 \\
\hline & 2 & 547 & 53 & 25 & 20,9 \\
\hline & total & 547 & 48 & 59 & 49,2 \\
\hline \multirow[t]{4}{*}{2} & i & 654 & 21 & 5 & 4,2 \\
\hline & 1 & 662 & 46 & 43 & 35,8 \\
\hline & 2 & 687 & 58 & 7 & 5,8 \\
\hline & total & 664 & 46 & 55 & 45,8 \\
\hline \multirow[t]{3}{*}{3} & 1 & 785 & 21 & 2 & 1,7 \\
\hline & 2 & 777 & 93 & 3 & 2,5 \\
\hline & total & 789 & 77 & 5 & 4,2 \\
\hline
\end{tabular}

Age de mer

L'âge de mer total des poissons capturés s'étale de 0+ à 3+. Les poissons ayant un séjour marin de $1+$ et $2+$ ans dominent très fortement et sont en proportion similaire, respectivement $49,2 \%$ et $45,8 \%$ (Tableau I).

L'AMM de l'échantillon est de 1,53 (écart type de la moyenne $=0,05$ ) et celui de la population de 1,54 (écart type de la moyenne $=0,06$ ).

La longueur totale moyenne des individus augmente des $0+$ de mer aux $3+$ de mer avec un accroissement d'environ $120 \mathrm{~mm}$ par année de séjour marin supplémentaire (Tableau I). 
Age de première reproduction et types de truite de mer

L'AMR (Age Moyen marin de première Reproduction) de l'échantillon est de 1,38 (écart type de la moyenne $=0,05$ ) et celui de la population de 1,39 (écart type de la moyenne $=0,06)$. Dans l'échantillon, quatre types de truite de mer ont pu être mis en évidence (Tableau II) largement dominés par le type I (61,7\% de l'échantillon), les types 0 et III étant quasiment inexistants.

\section{Tableau II}

Effectifs des différentes catégories d'un échantillon de 120 adultes de truite de mer capturés dans le Rhin en fonction de leur nombre d'hivers marins avant la première reproduction ( $i$ = âge d'eau douce indéterminé).

\section{Table II}

Numbers of different types of sea trout in a sample of 120 adults captured in the upper Rhine by sea age at first maturity ( $i=$ freshwater age unknown).

\begin{tabular}{|c|c|c|c|c|c|c|c|}
\hline \multirow{2}{*}{$\begin{array}{l}\text { Age marin à la } \\
\text { première reproduction }\end{array}$} & \multirow[t]{2}{*}{ Age d'eau douce } & \multicolumn{6}{|c|}{ Age de mer } \\
\hline & & 0 & 1 & 2 & 3 & total & $\%$ \\
\hline O & 1 & 1 & 0 & 0 & 0 & 1 & $0,8 \%$ \\
\hline \multirow[t]{4}{*}{1} & $\mathbf{i}$ & ----- & 4 & 1 & 0 & 5 & \\
\hline & 1 & ---- & 30 & 8 & 0 & 38 & \\
\hline & 2 & $-\cdots-\cdot$ & 25 & 3 & 3 & 31 & \\
\hline & total & 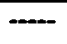 & 59 & 12 & 3 & 74 & $61,7 \%$ \\
\hline \multirow[t]{4}{*}{2} & $\mathbf{i}$ & $\cdots \cdots$ & $\cdots$ & 4 & 0 & 4 & \\
\hline & 1 & $\cdots-$ & $-\cdots$ & 35 & 0 & 35 & \\
\hline & 2 & $\cdots-.$. & ---- & 4 & 0 & 4 & \\
\hline & total & $-\cdots$ & ב-- & 43 & 0 & 43 & $35,8 \%$ \\
\hline 3 & 1 & $-\cdots$ & $\cdots$ & - & 2 & 2 & $1,7 \%$ \\
\hline
\end{tabular}

Compte tenu des faibles effectifs de certaines catégories de l'échantillon (Tableau II), l'influence de l'âge de première reproduction sur la croissance linéaire n'a pu être testée que sur les individus âgés de 1-2+. Dans ce groupe, les longueurs totales moyennes de $667 \mathrm{~mm}(\mathrm{n}=35 ; \sigma=48)$ et $642 \mathrm{~mm}(\mathrm{n}=8 ; \sigma=28)$ respectivement pour ceux qui se reproduisent pour la première fois à 1 et 2 ans de mer ne sont pas significativement différentes (Test de Student; $P=0,17$ ). II semble donc que la première reproduction n'ait que peu d'influence sur la croissance linéaire des individus, à même âge de smoltification et âge de mer à la capture.

L'examen des écailles révèle une faible proportion de poissons ayant déjà frayé (Tableau II). En effet, seuls 15 poissons sur $120(12,5 \%)$ se sont déjà reproduits une ou deux fois : 12 truites $2+$ de mer (avec une première reproduction à $1+$ de mer) et 3 truites $3+$ de mer (avec deux reproductions à $1+$ et $2+$ de mer).

\section{Liaisons entre les traits d'histoire de vie}

Les truites ayant smoltifié à 1 an sont majoritairement des 2+ ans de mer $(56,6 \%)$ alors que celles ayant smoltifié à 2 ans sont surtout des $1+$ de mer $(71,4 \%)$ (Figure 4). Ainsi les AMM (Ages Moyens de Mer) de 1,61 $(n=76 ; \sigma=0,54)$ et $1,37(n=35 ; \sigma=0,65)$ respectivement pour les poissons ayant smoltifié à 1 an et 2 ans sont statistiquement différents (Test $\mathrm{t}, \mathrm{P}=0,049$ ). 
1 an d'eau douce

$57 \%$

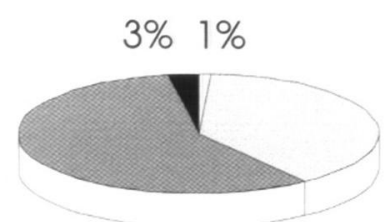

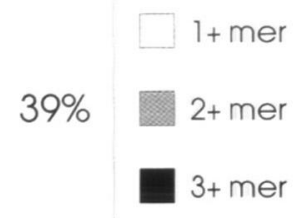

2 ans d'eau douce

O+ mer

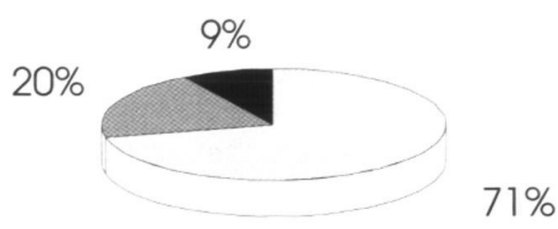

\section{Figure 4}

Ages de mer en fonction des âges de smoltification des adultes de truite de mer capturés dans le Rhin (échantilion de 76 truites âgées d'1+ an d'eau douce et 35 de 2+ ans d'eau douce).

\section{Figure 4}

Sea age and smolt age of adult sea trout captured in the Rhine (sample of 76 trout $1+$ smolt age and 35 trout $2+$ smolt age).

De même, il existe aussi une relation entre l'âge de smoltification et l'âge de mer de première reproduction. Les AMR (Ages Moyens de première Reproduction) sont de $1,50(\sigma=0,55)$ et $1,11(\sigma=0,32)$ respectivement pour les poissons ayant smoltifié à 1 an et 2 ans. Les truites ayant smoltifié à 2 ans se reproduisent statistiquement (Test $t$, $P<0,001)$ après un séjour marin plus bref que celles ayant smoltifié à 1 an.

Parmi les 1+ de mer, il y a la même proportion de truites qui ont passé 1 ou 2 ans en rivière, tandis que parmi celles de $2+$ de mer, la majorité n'a séjourné que 1 an en eau douce.

Un même phénomène est observé quand sont considérés les âges de première reproduction au lieu des âges de mer. On note aussi que pour les truites âgées de 3+ de mer, les trois s'étant reproduites après un hiver passé en mer ont toutes smoltifié à 2 ans alors que les deux qui se sont reproduites la première fois après trois hivers passés en mer ont smoltifié à 1 an.

\subsection{Evolution saisonnière des caractéristiques}

Le pourcentage d'individus âgés de $1+$ de mer augmente de juin à décembre (Figure 5), ce qui se traduit par une diminution de l'AMM alors que l'on se rapproche de la période de reproduction. En effet, les AMM de mai-juin et de novembre-décembre, respectivement égaux à $1,86(\sigma=0,38)$ et $1,36(\sigma=0,57)$, sont statistiquement différents (Test de Student ; $P=0,052$ ). Par ailleurs, de juin à décembre, le pourcentage de poissons ayant déjà frayé diminue (Figure 6).

II n'existe pas de différence significative de la taille moyenne mensuelle des poissons au cours de la période de migration anadrome bien qu'il y ait une tendance à leur diminution.

\section{DISCUSSION}

\subsection{Les limites méthodologiques de l'étude}

Quand l'échantillon des captures est réalisé par piégeage continu toute l'année (échantillonnage systématique) comme dans les cas de la Bresle, de la Touques et de 


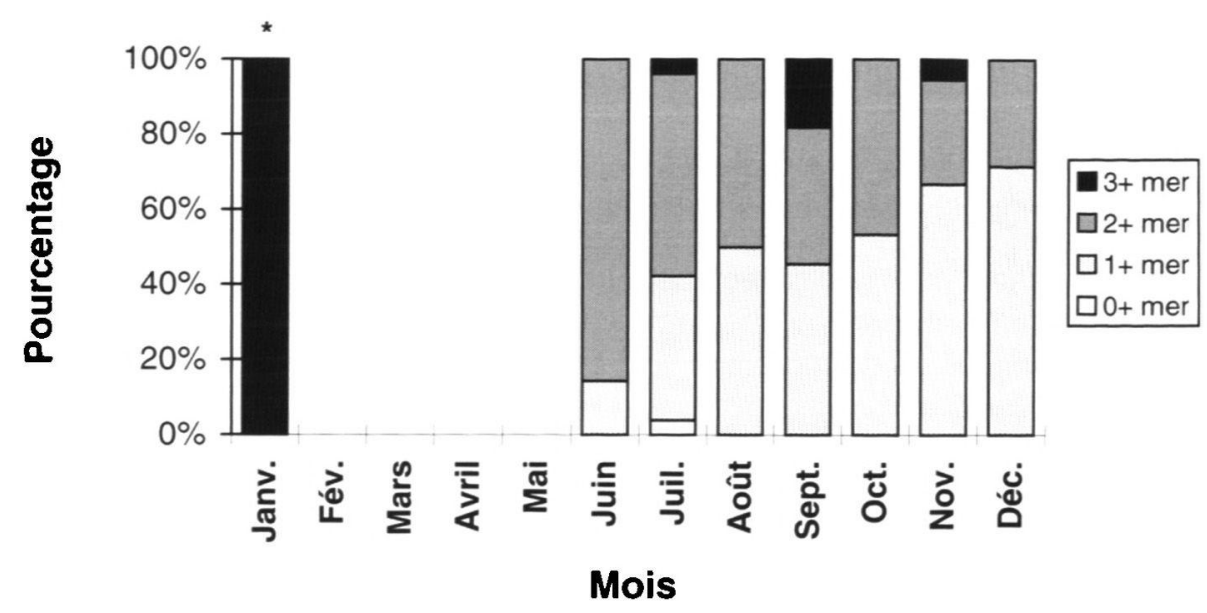

Figure 5

* = 1 seul individu canturé

Répartition mensuelle des captures des adultes de truite de mer du Rhin en fonction des âges de mer (échantillon de 118 truites dont la date de capture est connue).

Figure 5

Monthly distribution of catches of Rhine adult sea trout by sea age (sample of 118 trout with known catch date).

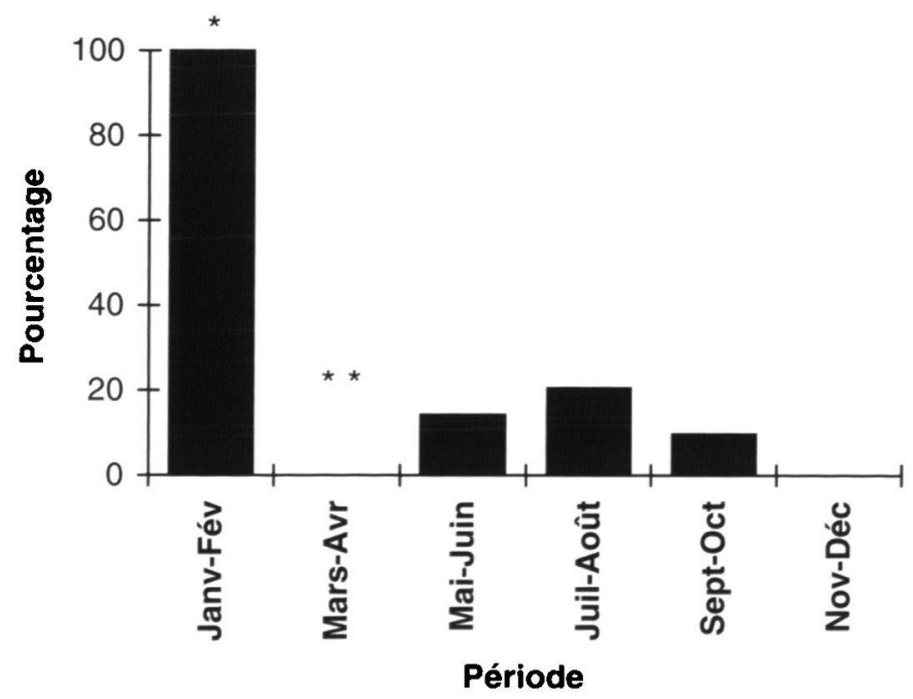

$$
\begin{aligned}
& * \quad=1 \text { seul individu capturé } \\
& * * \quad=0 \text { individu capturé }
\end{aligned}
$$

Figure 6

Evolution par période de 2 mois des pourcentages de truite de mer ayant déjà frayé (échantillon de 118 truites dont la date de capture est connue).

Figure 6

Evolution of repeat spawner percentage (sample of 118 trout with known catch date). 
l'Orne (EUZENAT et al., 1991), il est reconnu que les caractéristiques moyennes de cet échantillon sont représentatives de celles de la population migratrice totale. La méthodologie proposée ici a pour but d'estimer les caractéristiques biologiques de l'ensemble de la population présente dans le Rhin supérieur à partir des caractéristiques d'un échantillon capturé à différentes occasions pendant la période de migration anadrome afin de les comparer à celles d'autres réseaux hydrographiques. Cependant, cette méthode d'estimation nécessite que les caractéristiques moyennes mensuelles de la population arrivant et stationnant en aval du barrage d'lffezheim soient bien représentées par les caractéristiques moyennes mensuelles des captures. Pour cela, il a fallu émettre deux hypothèses dont il importe de discuter la portée.

La première hypothèse repose sur une capturabilité q constante des truites de mer par la pêche à l'électricité quels que soient la taille et l'état physiologique du poisson et quelle que soit la période de la sortie. Si l'équiprobabilité de capture des poissons en fonction de la taille est vraisemblable dans la gamme des cibles (446 à $770 \mathrm{~mm}$ de longueur totale), il semble que la capturabilité varie avec des phénomènes périodiques tels les conditions hydroclimatiques et le comportement du poisson en liaison avec son stade de maturation (ROCHE, 1996). En effet, lors des crues de fonte des neiges, avec de l'eau turbide, les truites se réfugient près des rives à une faible profondeur, ce qui facilite leur capture à l'électricité. Dès que les eaux s'éclaircissent, les captures deviennent plus rares. De même, en fin de période de migration, à l'approche de la période de reproduction, les truites bloquées sous les barrages apparaissent moins méfiantes et de ce fait plus facilement capturables quelles que soient les conditions de débits et de turbidité. Cependant, il a été montré que le modèle d'estimation des âges moyens était peu sensible à la variabilité mensuelle de la capturabilité. Donc, le fait que cette première hypothèse ne soit pas toujours vérifiée ne remet pas en cause le modèle proposé.

La seconde hypothèse suppose que les truites non capturées ne stationnent pas en aval du barrage d'lffezheim afin que l'échantillon capturé à une période donnée représente bien la population arrivant au barrage à cette même période. Dans la mesure où toutes les truites capturées sont marquées et relâchées en amont ou soustraites au milieu, l'erreur serait minime si la capturabilité était élevée. En l'absence de données sur la capturabilité, seules des observations de terrain permettent de discuter la validité de cette hypothèse. Des opérations de radiopistage (GERLIER et al., 1997) ont montré qu'une truite bloquée à Gambsheim avait rapidement dévalé vers l'embouchure d'un affluent accessible alors que I'on se rapprochait de la période de reproduction. En revanche, en période estivale, des saumons atlantiques sont restés deux mois en aval du barrage d'Iffezheim. Par ailleurs, les quelques sorties de pêche réalisées en aval du barrage de Gambsheim ont occasionné en 1996 des captures exclusivement de poissons non marqués. En 1994 et 1995, des captures de truites de mer y ont été faites alors que tous les poissons capturés à Iffezheim étaient soustraits du milieu. Ces observations laissent supposer que le franchissement des barrages par les écluses est loin d'être négligeable. De ce fait, le stationnement des truites de mer en aval du barrage d'Iffezheim peut être considéré comme limité par la recherche de zones de frai en aval et le franchissement possible de l'obstacle. Aussi, la seconde hypothèse émise est vraisemblable.

Cependant, ces diverses observations de terrain sur la variabilité de la capturabilité des truites de mer et sur leurs mouvements migratoires demanderaient à être confirmées par des opérations de capture-recapture voire de radiopistage afin d'améliorer la méthode de calcul.

Un autre problème rencontré lors de l'analyse des résultats est l'absence d'efforts de pêche pour les mois de janvier et février, et leur faiblesse de mars à mai. La répartition annuelle des efforts de pêche s'appuie sur le fait que la migration anadrome des truites de mer ne commence qu'au printemps dans le cours aval du Rhin au Pays Bas (CAZEMIER, 
1992) et sur des rivières proches (la Bresle en Picardie ; EUZENAT et al., 1991). De fait, les quelques CPUE qui ont pu être calculées pour les mois de mars, avril et mai sont nulles. Bien que peu probables étant donné la durée et la distance de la migration, il serait cependant nécessaire de vérifier qu'il n'y a pas de remontées tardives en janvier et février comme cela a été observé sur certains fleuves côtiers sédimentaires (EUZENAT et al., 1991).

Les différents âges moyens caractérisant les truites de mer du Rhin supérieur, calculés pour la population (utilisation des CPUE) et pour l'échantillon, ne montrent pas de différences significatives. II n'y a pas non plus d'augmentation de la précision de ces âges moyens. Ceci est vraisemblablement dû au fait que l'échantillon est composé pour presque la moitié (55 individus sur 120) de poissons capturés en 1996 et que l'évolution des CPUE mensuelles de cette même année est comparable à celle des CPUE moyennes pour la période 1994-1996.

Enfin, l'hétérogénéité de la répartition annuelle des échantillons d'écailles ne permet pas de comparaisons interannuelles des caractéristiques moyennes du stock, ce qui aurait pu permettre une certaine validation de l'utilisation des CPUE pour l'estimation de ces caractéristiques.

\subsection{Rythme de migration}

Comme pour la Bresle, la Touques et l'Orne (EUZENAT et al., 1991), la migration des truites de mer du Rhin se déroule en deux vagues successives. Par contre, les pics de migration (juillet-août et novembre) comme la période creuse intermédiaire (septembre-octobre) sont décalés d'environ un mois. Ceci peut s'expliquer par le fait que l'on compare des rythmes de migration dans les parties aval de cours d'eau picards et normands à ceux enregistrés à environ $700 \mathrm{~km}$ de la mer pour le Rhin. Par ailleurs, les poissons à long séjour marin et à frais multiples du Rhin migrent préférentiellement en début de saison comme pour beaucoup d'autres cours d'eau (LE CREN, 1985 ; EUZENAT et al., 1991).

\subsection{Caractéristiques des adultes de truite de mer du Rhin supérieur}

L'analyse scalimétrique de l'échantillon de la forme migratrice de la truite commune (Salmo trutta L.) capturée dans le Rhin donne un AMS, Age Moyen de Smoltification (âge moyen d'eau douce), de 1,31 an. Un départ en mer après un court séjour en eau douce rapproche les truites de mer du Rhin de celles de l'Orne, de la Touques et de la Bresle qui se jettent dans la Manche (EUZENAT et al., 1991), mais les distingue de celles des cours d'eau de la façade atlantique (Tableau III) ou de cours d'eau plus nordiques (JONSSON et L'ABÉE-LUND, 1993) caractérisés par un AMS plus élevé. Notamment l'AMS de la population de la Vistule, grand fleuve comparable au Rhin dont l'estuaire se situe plus au nord à une latitude de $54^{\circ} \mathrm{N}$, a été estimé à 2,08 (ZARNECKI, 1960 in RICHARD, 1986).

La structure d'âge de mer total de l'échantillon de truites de mer du Rhin est peu étalée, de $0+$ à $3+$, avec une prédominance partagée des groupes $1+(48,8 \%)$ et $2+$ $(46,3 \%)$ de mer. Cette structure est beaucoup plus proche de celle de l'Orne que de celle de la Bresle ou de la Touques (RICHARD, 1986 ; EUZENAT et al., 1991). En effet, sur l'Orne, il y a $43 \%$ de type $1+$ et $33 \%$ de $2+$ contre $75 \%$ de $1+$ et $15 \%$ de $2+$ sur la Bresle ou même les $20 \%$ de $0+$ et $72 \%$ de $1+$ sur la Touques. Le spectre d'âge de mer réduit rapproche le Rhin des cours d'eau dans lesquels les truites ont un taux de croissance élevé telle la Vistule (ZARNECKI, 1960 in RICHARD, 1986) ou des conditions d'accès aux frayères difficiles (BACHELIER, 1966 in RICHARD, 1986). L'existence probable d'un finnock dans l'échantillon analysé demanderait à être vérifiée par le dosage du strontium notamment, qui permettrait peut être de préciser si le poisson a rejoint la mer ou s'il a seulement atteint les lacs estuariens hollandais (ROCHE, 1992). En effet, aucune structure 


\section{Tableau III}

Age Moyen de Smoltification (AMS) et Age Moyen de mer à la première Reproduction (AMR) pour quelques rivières européennes calculés à partir d'échantillons de $n$ individus smolts (S) ou adultes (A). D'après (1) BAGLINIÈRE, 1979 ; (2) EUZENAT et al., 1991 ; (3) JONSSON et L'ABÉE-LUND, 1993 ; (4) PROUZET et al., 1989, 1992, 1994 ; (5) RICHARD, 1986 ; (6) TOLEDO et al., 1993.

\section{Table III}

Mean smolting age (AMS) and mean sea age at first maturity (AMR) for a few European rivers calculated with samples of $n$ individuals smolt (S) or adult (A). From (1) BAGLINIÈRE, 1979 ; (2) EUZENAT et al., 1991 ; (3) JONSON and L'ABÉE-LUND, 1993 ; (4) PROUZET et al., 1989, 1992, 1994 ; (5) RICHARD, 1986 ; (6) TOLEDO et al., 1993.

\begin{tabular}{|c|c|c|c|c|c|}
\hline Rivières de la façade atlantique & Latitude nord & AMS & (n) & AMR & (n) \\
\hline Carès (Asturies) (6) & $4330^{\prime}$ & 2,03 & (159 A) & 0,24 & $(159 \mathrm{~A})$ \\
\hline Adour (4) & $43^{\prime} 30^{\prime}$ & 1,81 & $(264 \mathrm{~A})$ & 0,35 & $(264 A)$ \\
\hline Ellée (1) & 47.45 & 1.93 & $(58 \mathrm{~S})$ & & \\
\hline \multicolumn{6}{|l|}{ Rivières de la Manche } \\
\hline Touques (2) (5) & $49^{\prime} 15^{\prime}$ & 1,45 & $(>3500 \mathrm{~A})$ & 0,89 & $(>3500 \mathrm{~A})$ \\
\hline Bresle (2) & $50^{-}$ & 1,22 & $(>30005)$ & 1,05 & $(>4000 \mathrm{~A})$ \\
\hline Orne (5) & $49^{\circ} 15^{\prime}$ & 1,15 & $(>2000 \mathrm{~A})$ & 1,33 & $(>2000 \mathrm{~A})$ \\
\hline Rhin & $52^{\circ}$ & 1,30 & $(111 \mathrm{~A})$ & 1,39 & $(120 \mathrm{~A})$ \\
\hline \multicolumn{6}{|l|}{ JONSSON et L'ABÉE-LUND (1993) } \\
\hline Rivières en moyenne & $54^{\circ}$ & 2,1 & & 0,5 & \\
\hline Rivières en moyenne & $70^{\circ}$ & 4,5 & & 2,7 & \\
\hline
\end{tabular}

telle celle définie par RICHARD et BAGLINIĖRE (1990) traduisant une incursion en milieu estuarien ou dulçaquicole après un court séjour en mer (inférieur à 6 mois) n'a été observée sur les écailles des truites âgées de plus d'un an de mer. Quoi qu'il en soit, le faible pourcentage de finnocks trouvé est en accord avec la structure d'âge de mer des truites anadromes sur des grands cours d'eau comme la Vistule (ZARNECKI, 1960) ou sur d'autres rivières de moindre importance comme l'Orne (RICHARD, 1986) ou la Tweed en Ecosse (PATERSON, 1973).

Les truites de mer du Rhin présentent de fortes croissances, supérieures même à celles observées sur la Vistule (ZARNECKI, 1960) pourtant considérées comme de gros individus comparativement à de nombreuses rivières européennes (RICHARD, 1986). Les poissons âgés de $1+$ de mer mesurent en moyenne $55 \mathrm{~cm}$ et $50 \mathrm{~cm}$ respectivement sur le Rhin et la Vistule. Les individus de 2+ de mer sont quant à eux dans des gammes de taille de $53-79 \mathrm{~cm}$ ( $66 \mathrm{~cm}$ en moyenne) et de $65-70 \mathrm{~cm}$ respectivement pour ces deux cours d'eau.

La composition typologique basée sur l'âge de première reproduction montre une prédominance du type $\mid(61,7 \%$ de l'échantillon) et de très faibles effectifs des types 0 $(0,8 \%)$ et III $(1,8 \%)$. L'AMR, Age Moyen de première Reproduction, du Rhin $\left(52^{\circ} \mathrm{N}\right)$ est supérieur ou équivalent aux AMR calculés sur certains cours d'eau français (Tableau III) de latitude équivalente tels la Bresle $\left(50^{\circ} \mathrm{N}\right)$ et l'Orne $\left(49^{\circ} 15^{\prime} \mathrm{N}\right)$. Comparé aux résultats de JONSSON et L'ABÉE-LUND (1993) qui ont montré que l'AMR augmentait avec la latitude et passait en moyenne de $0,4\left(54^{\circ} \mathrm{N}\right)$ à $2,7\left(70^{\circ} \mathrm{N}\right)$, l'AMR du Rhin est élevé. Ceci peut s'expliquer par le fait que l'AMR dépend, outre de la position latitudinale du réseau 
hydrographique, de son importance (sites de reproduction éloignés de l'estuaire). En effet, l'AMR augmente de 0,89 à 1,39 quand on considère, à latitudes comparables, la succession des cours d'eau suivants par ordre croissant d'importance du réseau : la Touques, la Bresle, l'Orne et le Rhin. L'ABÉE-LUND (1991) a en effet montré que la taille (pour partie en relation avec l'âge) des truites anadromes augmentait avec les distances à parcourir en eau douce pour atteindre les sites de reproduction.

L'analyse des écailles révèle une faible proportion de poissons ayant déjà frayé : seulement $12,5 \%$ se sont déjà reproduits une ou deux fois. Ce pourcentage est comparable à celui observé sur la Touques (12\%) ou la Bresle (10 à $25 \%$ ) mais inférieur à celui de l'Orne (25 à $40 \%)$.

Les comparaisons des caractéristiques des truites de mer du Rhin avec celles d'autres cours d'eau reposent sur l'hypothèse que les structures des écailles ont été interprétées de la même manière par les différents chercheurs pour l'estimation des âges. Compte tenu de la grande diversité des structures et des difficultés d'interprétation des écailles de truite de mer (RICHARD et BAGLINIĖRE, 1990), cette hypothèse n'est bien entendu pas vérifiée. Cependant, du fait qu'une concertation ait eu lieu au niveau français dans le cadre du GPC (Groupe Permanent de Concertation) Amphihalins (nouvellement GRISAM), les ordres de grandeur des divers indices populationnels de la plupart des stocks sont " très vraisemblablement " comparables.

\subsection{Liaisons entre les traits d'histoire de vie}

Pour l'échantillon de truites de mer capturées sur le Rhin supérieur, l'âge de mer des captures augmente quand l'âge de smoltification diminue. Ce phénomène a déjà été observé sur la Touques (d'après les données de RICHARD, 1986). De même, l'âge marin à la première reproduction des truites de mer du Rhin augmente quand l'âge de smoltification diminue. Cela se traduit par le fait que respectivement $30,2 \%, 60,4 \%$ et $5,4 \%$ des truites de mer se reproduisent à un âge total (eau douce et mer confondues) de 2, 3 et 4 ans (Tableau II).

Par ailleurs, JONSSON et L'ABÉE-LUND (1993) ont montré pour différents stocks (102 rivières à des latitudes comprises entre 54 et $70^{\circ} \mathrm{N}$ ) que l'âge de mer moyen à la première maturité (AMR) était lié négativement à la taille moyenne des smolts mais par contre positivement à l'Age Moyen de Smoltification (AMS). Ceci va à l'encontre des observations faites sur des rivières françaises (dont le Rhin) et espagnoles (Tableau III). Aux latitudes les plus faibles de la répartition originelle de la truite Salmo trutta, l'AMR augmente avec une diminution de l'AMS.

\section{CONCLUSION}

En l'absence d'un système de comptage et de piégeage qui permettrait un suivi presque exhaustif, les captures de truites de mer par pêche à l'électricité représentent un palliatif utile pour récolter des données sur la population remontant dans le Rhin supérieur. Ce suivi sera amélioré prochainement avec l'utilisation d'un système couplant une caméra et un piège, prévu avec l'aménagement des passes à poissons sur les barrages d'Iffezheim et de Gambsheim. II sera particulièrement intéressant et utile de savoir si la méthode d'estimation des caractéristiques de la population du Rhin présentée dans ce travail sera validée par les données issues de cette méthode d'échantillonnage plus efficace.

Les indices moyens calculés pour le stock de truites de mer adultes du Rhin caractérisent probablement un ensemble hétérogène d'individus (origines naturelle et de déversement, différentes souches, divers milieux de production des juvéniles migrants...) 
dont les contributions respectives ne sont pas quantifiées. Mais ce fait concerne tant le Rhin que tous les autres cours d'eau auxquels il a été comparé, notamment parce que des déversements de juvéniles de truite sont systématiques au niveau national. Les caractéristiques moyennes des populations d'adultes intègrent donc cette hétérogénéité qui est cependant prise en compte dans les travaux sur le déterminisme (génétique, environnemental...) du phénomène de migration chez la truite commune (OMBREDANE et al., 1996).

Les caractéristiques de la population des truites de mer du Rhin déterminées par ce travail sont néanmoins typiques de celles de grands fleuves avec notamment des individus de gros gabarit et d'âge de première reproduction élevé pour la latitude.

\section{REMERCIEMENTS}

Les pêches à l'électricité ont été mises en oeuvre conjointement par l'Association Saumon-Rhin, la Fédération de Pêche du Bas-Rhin et le Conseil Supérieur de la Pêche. Pour la partie allemande de l'ouvrage d'Iffezheim (usine hydroélectrique), les pêches ont eu lieu avec la participation d'un représentant du Regierungspresidium de Karlsruhe. Ce travail d'interprétation des données a été cofinancé par l'Union Européenne et le Conseil Supérieur de la Pêche.

\section{BIBLIOGRAPHIE}

ANONYME, 1994. Saumon 2000. Commission Internationale pour la Protection du Rhin, $29 \mathrm{p}$. BACHELIER R., 1966. Le saumon polonais. Bull. Fr. Piscic., 220, 101-108.

BAGLINIÈRE J.L., 1979. Dévalaison de truites (Salmo trutta) sur la rivière Ellé. Bull. Fr. Piscic., 275, 49-60.

BAGLINIĖRE J.L., LE LOUARN H., 1987. Caractéristiques scalimétriques des principales espèces de poissons d'eau douce de France. Bull. Fr. Pêche Piscic., 306, 1-39.

CAZEMIER W.G., 1988. Fish and their environment in large European river ecosystems. The Dutch part of the River Rhine. Rev. des Sciences de l'Eau, 7 (1), 95-114.

CAZEMIER W.G., 1992. The migration of sea trout (Salmo trutta trutta L.) along the Dutch coast and in the lower part of the Rhine. Report 92-501, RIVO-DLO, IJmuiden, $27 \mathrm{p}$. + tableaux.

EUZENAT G., FOURNEL F., RICHARD A., 1991. La truite de mer (Salmo trutta L.) en Normandie/Picardie. In BAGLINIĖRE J.L. et MAISSE G. (eds), La truite, biologie et écologie, 183-213, INRA Editions, Paris.

FAHY E., 1978. Variation in some biological characteristics of British sea trout, Salmo trutta. J. Fish. Biol., 13, 123-138.

GERLIER M., ROCHE P., EDEL G., 1997. Etude par radiopistage de la migration de saumons et truites de mer adultes dans le bassin rhénan alsacien. Résultats 1996. Rapport CSP Metz-ASR Strasbourg, 23 p. + annexes.

GROOT S.J. (de), 1989. Literature survey into the possibility of restocking the River Rhine and its tributaries with sea trout (Salmo trutta trutta). RIVO, IJmuiden, Publication and reports. Vol. 1989-12 of the project "Ecological Rehabilitation of the River Rhine ", $53 \mathrm{p}$.

JONSSON B., L'ABÉE-LUND J.H., 1993. Latitudinal clines in life-history variables of anadromous brown trout in Europe. J. Fish Biol., 43 (supplément A), 1-16.

L'ABÉE-LUND J.H., 1991. Variation within and between rivers in adult size and sea age at maturity of anadromous brown trout, Salmo trutta. Can. J. Fish. Aquat. Sci., 48, 1015-1021. 
LAUREC A., LE GUEN J.C., 1981. Dynamique des populations exploitees - Tome I, concepts et modèles. CNEXO Rapports scientifiques et techniques $n^{\circ} 45$, Brest, $117 \mathrm{p}$.

LE CREN E.D., 1985, The biology of sea trout - Summary of a symposium held at Plas Menai, 24-26 October 1984. Atlantic Salmon Trust, 42 p.

OLBRICH P., 1984. Untersuchungen zum Wiedererscheinen der Meerforelle (Salmo trutta L.) im oberen Niederrheingebiet. Der Fischwirt, 33 (3), 22-24.

OMBREDANE D., RICHARD A., 1990. Détermination de la zone optimale de prélèvement d'écailles chez les smolts de truite de mer. Bull. Fr. Pêche Piscic., 319, 224-238.

OMBREDANE D., SIEGLER L., BAGLINIĖRE J.L., PRUNET P., 1996. Migration et smoltification des juvéniles de truite (Salmo trutta) dans deux cours d'eau de Basse-Normandie. Cybium, 20 (3) suppl., 27-42.

PATERSON D., 1973. Observations on the sea trout (Salmo trutta L.) spawning populations from eight Tweed tributaries. B. Sc. Thesis, University of Edinburgh, $110 \mathrm{p}$.

PHILIPPART J.C., 1983. Note sur la redécouverte de " truites de mer " dans un affluent de la Meuse liégeoise en 1983. Cahiers d'Ethologie appliquée, 3 (1), 105-114.

PROUZET P., MARTINET J.P., CASAUBON J., 1989. La Pêche des marins pêcheurs dans l'estuaire de l'Adour en 1988. Rapport IFREMER-INRA, St-Pée-sur-Nivelle, 17 p.

PROUZET P., MARTINET J.P., LURO C., 1992. La Pêche des marins pêcheurs dans l'estuaire de l'Adour en 1991. Rapport IFREMER-INRA, St-Pée-sur-Nivelle, $24 \mathrm{p}$.

PROUZET P., MARTINET J.P., CUENDE F.X., 1994. La Pêche des marins pêcheurs dans l'estuaire de l'Adour en 1993. Rapport IFREMER-INRA, St-Pée-sur-Nivelle, 19 p.

RICHARD A., 1986. Recherches sur la truite de mer Salmo trutta L. en Basse-Normandie. Thèse de Doctorat, Rennes, $54 \mathrm{p}$.

RICHARD A., BAGLINIĖRE J.L., 1990. Description et interprétation des écailles de truites de mer Salmo trutta $L$. de deux rivières de Basse-Normandie : l'Orne et la Touques. Bull. Fr. Pêche Piscic., 319, 239-257.

ROCHE P., 1992. Mise en évidence de l'écotype truite de mer dans les captures de grandes truites (Salmo trutta L.) du Rhin supérieur. Bull. Fr. Pêche Piscic., 324, 36-44.

ROCHE P., 1996. Restauration des écosystèmes aquatiques du bassin du Rhin, objectif saumon. Rapport CSP Metz-DIREN Alsace, $150 \mathrm{p}$.

ROCHE P., EDEL G., GERLIER M., 1996. Premières captures de saumons (Salmo salar L.) dans le Rhin franco-allemand et mise en évidence de frayères dans la Bruche. Rapport CSP Metz, $7 \mathrm{p}$.

SCHERRER B., 1983. Techniques de sondage en écologie. In FRONTIER S., Stratégies d'échantillonnage en écologie, 63-162, Collection d'écologie 17, Masson (éd.), Paris, $492 \mathrm{p}$.

STEINBERG L., LUBIENIECKI B., 1991. Die Renaissance der Meer-forelle (Salmo trutta trutta L.) und erste Versuche zur Wiedereinburgerung des Lachses (Salmo salar L.) in Nordrhein-Westfalen. Fischökologie, 5, 19-33.

STEINBERG L., MARMULLA G., SCHMIDT G.W., LEHMANN L., 1991. Erster gesicherter Nachweis des Laches Salmo salar L. in den Rhein Nebenfluss Sieg. Fischökologie, 5 (Teil 1), 3-18.

TOLEDO M. (del Mar), LEMAIRE A.L., BAGLINIĖRE J.L., BRAÑA F., 1993. Caractéristiques biologiques de la truite de mer (Salmo trutta L.) au nord de l'Espagne, dans deux rivières des Asturies. Bull. Fr. Pêche Piscic., 330, 295-306

WEIBEL U., 1991. Neue Ergebnisse zur Fischfauna des nordlichen Oberrheinsermittelt im Rechengut von Kraftwerken. Fischökologie, 5, 43-68.

ZARNECKI S.S., 1960. General conclusions on the scale-reading of salmon, sea trout and brown trout originating in the Vistula. ICES ANACAT, Fish. Comm., 128, 1-3. 